EGU21-15707

https://doi.org/10.5194/egusphere-egu21-15707

EGU General Assembly 2021

(c) Author(s) 2022. This work is distributed under

the Creative Commons Attribution 4.0 License.

\title{
Why switchbacks may be related to solar granulation
}

Naïs Fargette ${ }^{1}$, Benoit Lavraud ${ }^{1,2}$, Alexis Rouillard ${ }^{1}$, Victor Réville ${ }^{1}$, Tai Phan ${ }^{3}$, Stuart D. Bale ${ }^{3}$, Thierry Dudok De Wit ${ }^{4}$, Clara Froment ${ }^{4}$, Justin Kasper ${ }^{5}$, Jasper S. Halekas ${ }^{6}$, Philippe Louarn ${ }^{1}$, Anthony W. Case ${ }^{7}$, Kelly E. Korreck ${ }^{7}$, Davin E. Larson ${ }^{3}$, David Malaspina ${ }^{8,9}$, Marc Pulupa ${ }^{3}$, Michael L. Stevens ${ }^{7}$, Phyllis L. Whittlesey ${ }^{3}$, and Matthieu Berthomier ${ }^{10}$

${ }^{1}$ Institut de Recherche en Astrophysique et Planétologie, CNRS, UPS, CNES, Toulouse, France (nais.fargette@irap.omp.eu)

${ }^{2}$ Laboratoire d'Astrophysique de Bordeaux, CNRS, Bordeaux, France

${ }^{3}$ Space Sciences Laboratory, University of California, Berkeley, Berkeley, CA, USA

${ }^{4}$ LPC2E, CNRS and University of Orléans, Orléans, France

${ }^{5}$ Climate and Space Sciences and Engineering, University of Michigan, Ann Arbor, MI, US

${ }^{6}$ Department of Physics and Astronomy, University of lowa, lowa City, lowa, United States

${ }^{7}$ Smithsonian Astrophysical Observatory, Cambridge, Massachusetts, US

${ }^{8}$ Department of Astrophysical and Planetary Sciences, University of Colorado, Boulder, CO, USA

'Laboratory for Atmospheric and Space Physics, University of Colorado, Boulder, CO, USA

${ }^{10}$ Laboratoire de Physique des Plasmas, CNRS, Sorbonne Universite, Ecole Polytechnique, Observatoire de Paris, Universite

Paris- Saclay, Paris, France

Parker Solar Probe data below 0.3 AU have revealed a near-Sun magnetic field dominated by Alfvénic structures that display back and forth reversals of the radial magnetic field. They are called magnetic switchbacks, they display no electron strahl variation consistent with magnetic field foldings within the same magnetic sector, and are associated with velocity spikes during an otherwise calmer background. They are thought to originate either at the photosphere through magnetic reconnection processes, or higher up in the corona and solar wind through turbulent processes.

In this work, we analyze the spatial and temporal characteristic scales of these magnetic switchbacks. We define switchbacks as a deviation from the parker spiral direction and detect them automatically through perihelia encounters 1 to 6 . We analyze the solid angle between the magnetic field and the parker spiral both over time and space. We perform a fast Fourier transformation to the obtained angle and find a periodical spatial variation with scales consistent with solar granulation. This suggests that switchbacks form near the photosphere and may be caused, or at least modulated, by solar convection. 